Rezeptors 9 verbirgt, weiterverfolgt. Eine Phase-II-Studie mit Patienten mit metastasiertem CRC und Krankheitskontrolle nach Chemotherapie hatte insbesondere bei denjenigen mit partiellem Ansprechen auf die Induktionstherapie einen günstigen Effekt der Impfung auf das PFS im Vergleich zu Placebo gezeigt [Schmoll HJ et al. J Cancer Res Clin Oncol. 2014; 140 (9): 1615 - 24].
Standardempfehlungen für die Deeskalation der Therapie können derzeit nicht ausgesprochen werden. „Die Wahl der Erhaltungsstrategie sollte die Patientenpräferenz berücksichtigen“, betonte Hegewisch-Becker und sagte in Bezug auf Therapiepausen: „Wir können den Patienten vielleicht eine schöne Reise genehmigen, aber ich glaube nicht dass eine Weltreise möglich ist.“

\title{
Ernährungstherapie bei Tumorpatienten
}

\author{
Bei Patienten mit fortgeschrittener Tumorerkrankung \\ unter onkologischer Therapie sind die Erfassung und \\ konsequente Behandlung einer Mangelernährung \\ von erheblicher Bedeutung für das klinische Behand- \\ lungsergebnis und die Lebensqualität.
}

Nahrungsaufnahme ist für alle Menschen wichtig - nicht nur, um den Organismus zu erhalten: „Essen und Trinken haben auch kulturell und gesellschaftlich eine große Bedeutung. So werden Einladungen oft zum Essen ausgesprochen, man kocht zusammen, und auch in vielen Religionen steht das Mahl im Mittelpunkt ritueller Handlungen“, erläuterte Prof. Karin Oechsle, Universitätsklinikum Hamburg-Eppendorf.

Gerade bei Krankheit, Stress und in der Rekonvaleszenz sei eine gute Ernährung wichtig. „Das ist den meisten Menschen bewusst, und deshalb ist es für Krebspatienten oft sehr belastend, wenn sie zwar gerne essen möchten, es aber nicht können. Die Angehörigen fühlen sich zudem hilflos, weil sie dem Patienten das Essen gerne ermöglichen würden“, betonte Oechsle.

\section{Malnutrition und Tumorkachexie}

Von Mangelernährung sind zwischen $40 \%$ und $80 \%$ der onkologischen Patienten betroffen. Sie ist charakterisiert durch eine mangelnde Zufuhr an Energie, Proteinen, Vitaminen und Spurenelementen, die infolge von Funktionsstörungen des Gastrointestinaltrakts wie Mukositis, Schluckstörungen, Übelkeit oder Schmerzen entsteht. „Die Konsequenz der verminderten Energieaufnahme ist primär ein Fettverlust“, erläuterte Oechsle. Dagegen sei die Tumorkachexie, die primär zu einem Muskelverlust führe, eine Stoffwechselstörung: „Sie wird durch den Tumor selbst verursacht, geht oft mit Entzündungsreaktionen und einem erhöhten Grundumsatz einher“, erklärte die Palliativmedizinerin.

\section{Entscheidungsfindung nach Krankheitssituation}

Vor der Entscheidung, ob eine Ernährungstherapie vorgenommen wird, sollte eine Abklärung der Ursache für die Malnutrition oder Tumorkachexie stehen: Gibt es physikalische Behinderungen oder Appetitlosigkeit, die verhindern, dass der Patient isst und trinkt? Ist es der dezidierte Wille des Patienten, keine Nahrung aufzunehmen? Wie sind Prognose und Krankheitsdynamik - gibt es onkologische Therapieoptionen? Was ist das Ziel der Behandlung - Beseitigung von Mangelernährung und Kachexie, Erfüllung von Erwartungen beim Patienten oder den Angehörigen oder Lindern eines Leidensdrucks beim Pati- enten? „Letztendlich entscheidend ist, was der Patient selbst möchte“, sagte Oechsle. Wichtig für die Entscheidung, ob und welche therapeutischen Maßnahmen ergriffen werden, sei zudem die konkrete Erkrankungssituation: Ist die Kachexie reversibel? Oder ist die Prognose des Patienten mit weniger als vier Wochen limitiert und die Kachexie refraktär?

Säulen der Kachexietherapie sind Nährstoffzufuhr, Beratung und nicht zuletzt körperliche Aktivität. Einer oralen Therapie sollte stets der Vorzug vor der enteralen und der parenteralen Therapie gegeben werden. Medikamentöse Appetitsteigerung ist bei Bedarf möglich, eine psychologisch-seelsorgerische Unterstützung kann unterstützend wirken. In der letzten Lebensphase sollte nur noch eine symptomorientierte Ernährungstherapie vorgenommen werden. „Es ist definitiv ärztliche Aufgabe, dem Thema Ernährung Aufmerksamkeit zu schenken“, betonte Oechsle abschließend.

Dr. Silke Wedekind

\section{Pankreas-Ca: Noch immer kein Standard für perioperative Therapie}

Während der DGHO-Jahrestagung wurde für die Teilnahme an Studien zur perioperativen Therapie beim Pankreaskarzinom geworben.

Bei Patienten mit einem lokalen duktalen Adenokarzinom des Pankreas (PDAC) ist nach Ansicht von Prof. Volker Kunzmann, Universitätsklinikum Würzburg, ein präzises prätherapeutisches Staging essenziell. „Jeder Patient mit lokalisiertem oder lokal fortgeschrittenem PDAC soll in einem Tumorboard interdisziplinär besprochen werden", erklärte Kunzmann. Eine Prognoseverbesserung sei nur durch eine Optimierung oder eine Intensivierung der perioperativen Systemtherapie mit dem Ziel der R0-Resektion zu erwarten. Dies hätten die Ergebnisse der ESPAC-Studien klar gezeigt. In der aktuellen ESPAC-4-Studie etwa mit PDAC-Patienten nach R0- beziehungsweise R1-Resektion wurde die Wirksamkeit der Kombination aus Gemcitabin plus Capecitabin mit der einer Gemcitabin-Monotherapie verglichen - mit einem Vorteil beim Gesamtüberleben durch die Kombinationstherapie.

"Wir haben nach wie vor keinen Standard für die perioperative Therapie", betonte Kunzmann. Das betreffe sowohl die Art und die Dauer als auch die Sequenz der Behandlung. Deswegen empfahl er den Kollegen, die derzeit dazu laufenden Studien in Deutschland zu unterstützen. Im Fokus steht zum einen die NEONAX-Studie mit Patienten, die an einem resektablen Pankreaskarzinom erkrankt sind, in der ein neoadjuvanter Ansatz mit Gemcitabin/nab-Paclitaxel geprüft wird. Zum anderen läuft die NEOLAPStudie, an der Patienten mit lokal fortgeschrittenem Pankreaskarzinom teilnehmen, in der auch der Nutzen des FOLFIRINOX-Regimes und eine explorative Laparotomie geprüft werden.

Peter Leiner 